ISSN 0103-5150

Fisioter. Mov., Curitiba, v. 24, n. 2, p. 247-254, abr./jun. 2011

Licenciado sob uma Licença Creative Commons

\title{
Ganho de extensibilidade dos músculos isquiotibiais comparando o alongamento estático associado ou não à crioterapia
}

\author{
Hamstrings muscles extensibility gain comparing static stretching \\ with or without cryotherapy
}

\section{Fernanda de Oliveira Busarello ${ }^{[a]}$, Francieli Tibes de Souza ${ }^{[b]}$, Grazieli Francine de Paula ${ }^{[c]}$, Lizyana Vieira $^{[\mathrm{d}]}$, Gustavo Kiyosen Nakayama ${ }^{[\mathrm{el}}$, Gladson Ricardo Flor Bertolini ${ }^{[\mathrm{fl}}$}

[a] Discente do curso de Fisioterapia da Universidade Estadual do Oeste do Paraná (Unioeste), Cascavel, PR - Brasil, e-mail: ferbusarello@hotmail.com

[b] Discente do curso de Fisioterapia da Universidade Estadual do Oeste do Paraná (Unioeste), Cascavel, PR - Brasil, e-mail: fran_ftds@hotmail.com

[c] Discente do curso de Fisioterapia da Universidade Estadual do Oeste do Paraná (Unioeste), Cascavel, PR - Brasil, e-mail: grazzifp@hotmail.com

[d] Discente do curso de Fisioterapia da Universidade Estadual do Oeste do Paraná (Unioeste), Cascavel, PR - Brasil, e-mail: lizyana@gmail.com

[e] Docente do curso de Fisioterapia da Universidade Estadual do Oeste do Paraná (Unioeste), Cascavel, PR - Brasil, e-mail: gknakayama@gmail.com

[f] Docente do curso de Fisioterapia da Universidade Estadual do Oeste do Paraná (Unioeste), Cascavel, PR - Brasil, e-mail: gladson_ricardo@yahoo.com.br

\section{Resumo}

Introdução: A crioterapia é indicada para diminuir o desconforto durante as sessões de alongamento e proporcionar melhores resultados no ganho de extensibilidade. Objetivo: 0 objetivo do estudo foi verificar o ganho de extensibilidade dos músculos isquiotibiais, comparando o alongamento estático com o alongamento estático associado à crioterapia. Materiais e métodos: Participaram 20 indivíduos, com idade entre 18 e 25 anos, divididos igualmente em dois grupos, os quais realizaram alongamento e alongamento associado à crioterapia. Foram utilizadas bolsas de gelo, na região posterior da coxa, durante 15 minutos. Em seguida, foi realizada a aplicação do alongamento estático de isquiotibiais, em duas séries, de 30 segundos cada. Resultados: Foram encontradas diferenças significativas na extensibilidade dos músculos isquiotibiais em ambos os grupos, porém não houve diferenças significativas na extensibilidade dos músculos isquiotibiais 
quando comparado o alongamento estático com o alongamento estático associado à crioterapia. Conclusão: Houve aumento imediato da extensibilidade dos músculos isquiotibiais. Porém, o uso de crioterapia não se mostrou eficaz para o ganho de extensibilidade.

Palavras-chave: Exercícios de alongamento muscular. Crioterapia. Maleabilidade.

\begin{abstract}
Introduction: Cryotherapy is indicated to decrease the discomfort during the sessions of stretching and provide better results in the gain of extensibility. Objective: Study's objective was to verify the gain extensibility of hamstring muscles, comparing the static stretching with static stretching associated with cryotherapy. Materials and methods: Twenty subjects participated of the study, aged between 18 and 25 years, divided equally in two groups, considering that both performed stretching and stretching associated with cryotherapy. It has been used ice packs on the back of the thigh for 15 minutes. Then, it has been realized the application of static stretching of hamstrings, in two sets of 30 seconds each. Results: It has been found significant differences in the extensibility of hamstring muscles in both groups, but there were no significant differences in the extensibility of hamstring muscles when compared static stretching with static stretching associated with cryotherapy. Conclusion: There was an immediate increase of the extensibility of hamstring muscles. However, the use of cryotherapy was not effective for gain of extensibility.
\end{abstract}

Keywords: Muscle stretching exercices. Cryotherapy. Pliability.

\section{Introdução}

A extensibilidade é tão importante para atletas quanto para pessoas sedentárias, uma vez que limitações na amplitude podem comprometer o desempenho esportivo, laboral ou de atividades diárias (1). Assim, alguns profissionais da saúde têm considerado o ganho de extensibilidade como sendo um componente integral na melhora no desempenho de atividades diárias e esportes (2).

Os exercícios de alongamento tendem a restabelecer níveis satisfatórios de mobilidade articular e reduzir tensões musculares, diminuindo dores e melhorando o desempenho muscular de atletas (1, 3-6). Assim, há uma contribuição para o treinamento de força e potência muscular (7).

Em relação à performance e ao alongamento, estudos que envolveram programas de alongamento e verificaram seus efeitos crônicos constataram que o alongamento realizado antes da prática esportiva ou do exercício trouxe ganhos para a flexibilidade e performance muscular em longo prazo (8-10).

0 alongamento é um exercício que aumenta o comprimento das estruturas dos tecidos moles e a flexibilidade pode ser considerada como a capacidade física responsável pela máxima amplitude de movimento (ADM), de uma ou mais articulações.
Ela deve ser livre de dor, sem restrições e sem o risco de lesão (1,11-13).

Alguns autores verificaram efeitos deletérios agudos quando se realiza um exercício de flexibilidade antes de atividades que utilizam força, pois foi observado um decréscimo na potência e força muscular. Isso foi verificado em estudos que utilizaram mais de um exercício de alongamento para o mesmo músculo, com permanência na posição por mais de 60 segundos e, principalmente, com realização de alongamentos estáticos (11,14-16).

Essa diminuição da força muscular após o alongamento pode estar relacionada com a inibição de mecanismos neurais, aumento da complacência e alterações nas propriedades viscoelásticas das unidades músculo-tendíneas e redução da ativação de unidades motoras (15-17).

Há controvérsias em relação à técnica, duração e frequência do alongamento, quando se propõe ganho de extensibilidade. A intensidade das tensões e a análise da participação dos componentes neurais e viscoelásticos durante o programa também têm sido estudados (18-21).

Estudos indicam que o alongamento sustentado por 30 segundos é suficiente para aumentar o comprimento muscular e, consequentemente, a ADM. Nenhum aumento ocorreu na extensibilidade 
quando a duração do alongamento foi aumentada de 30 para 60 segundos ou quando a frequência do alongamento foi aumentada de uma para três vezes por dia (5, 22-24).

Diversos tipos de alongamento foram desenvolvidos com o objetivo de aumentar a extensibilidade, como o alongamento ativo, passivo, balístico, facilitação neuromuscular proprioceptiva e alongamento estático $(4,25,26)$. Segundo Anderson e Burke (27), alongamento estático é definido como "tolerância ao alongamento do músculo sustentando uma posição por um determinado período de tempo". Esse alongamento é o mais utilizado para se obter aumento da extensibilidade e relaxamento muscular, além de se mostrar eficaz no aumento do comprimento muscular $(4,24,26,28)$. Assim, é considerado o padrão-ouro para o ganho de extensibilidade (4).

Buscar técnicas que aperfeiçoem os protocolos utilizados nas intervenções de alongamento está entre um dos grandes objetivos terapêuticos da atualidade (19). Para proporcionar melhores resultados na extensibilidade e diminuir o desconforto durante as sessões de alongamento, é sugerido, então, o uso prévio da termoterapia (29). Dentre as técnicas destaca-se a crioterapia, por reduzir a velocidade de condução nervosa e, consequentemente, diminuir a descarga fusal e a dor (19).

A crioterapia é utilizada para diminuir o edema, a inflamação, o fluxo sanguíneo, a taxa metabólica intramuscular, a hipertonicidade e a velocidade de condução nervosa (30). Estudos utilizando a aplicação de gelo ao longo do músculo, antes e durante o alongamento, verificaram um aumento significativo da extensibilidade dos isquiotibiais, sobre os resultados de grupos apenas com alongamento (25, 31). Porém, há controvérsias, pois Signori et al. (29) demonstraram que meios físicos de hipotermia e hipertermia aplicados previamente ao alongamento não influenciaram o aumento da extensibilidade dos músculos.

Além disso, o resfriamento, por reduzir a velocidade de condução nervosa, reduz a descarga fusal, diminuindo-se, por conseguinte, a interferência desse estímulo na tensão muscular (32). Contudo, não há um consenso de como executar o alongamento de forma adequada para garantir sua máxima eficiência. Em conjunto com o alongamento pode ser usada a crioterapia, para aumentar a amplitude de movimento. Porém, sua utilização ainda é incerta, visto que esta reduz a extensibilidade do colágeno, ou seja, do perimísio muscular (33).

Assim, o objetivo do presente estudo foi verificar o ganho de extensibilidade dos músculos isquiotibiais, comparando o alongamento estático com o alongamento estático associado à crioterapia.

\section{Materiais e métodos}

\section{Caracterização do estudo}

0 presente estudo trata-se de um ensaio clínico aleatório, por conveniência, estratificado, cruzado. Prévio ao início da pesquisa, o projeto foi aprovado pelo Comitê de Ética em Pesquisas em Humanos da Universidade Estadual do Oeste do Paraná (Unioeste), por meio do Processo $27371 / 2009$. Todos os participantes assinaram, de forma voluntária, o Termo de Consentimento Livre e Esclarecido quanto aos objetivos e procedimentos do estudo. Participaram 20 indivíduos, com idade entre 18 e 27 anos, de ambos os sexos. A amostra foi dividida em dois grupos, $A(n=10)$ e $B(n=10)$, e ambos realizaram alongamento e alongamento associado à crioterapia, porém, em dias diferentes. Os protocolos foram realizados no Laboratório de Estudo das Lesões e Recursos Fisioterapêuticos da Unioeste.

Como critérios de inclusão no estudo adotaram-se: ser acadêmico da instituição e apresentar extensibilidade de isquiotibiais menor que $160^{\circ}$ de extensão de joelho. Como critérios de exclusão foram utilizados: não comparecer em um dos dias da realização do tratamento ou da avaliação; possuir doenças músculoesqueléticas; apresentar hipersensibilidade ao frio; apresentar rigidez articular do joelho; modificar atividade física durante a intervenção, ou seja, se o indivíduo era ativo, deveria manter-se ativo, o mesmo acontecendo se fosse sedentário.

A avaliação da extensibilidade dos isquiotibiais foi realizada, por um avaliador cego, em todos os indivíduos no primeiro dia (AV1), seguido da divisão dos grupos, aleatoriamente. No segundo dia foi realizada novamente avaliação nos dois grupos, antes (AV2) e depois (AV3) da intervenção, que consistiu de: grupo A - alongamento estático de isquiotibiais; e grupo B - alongamento estático de isquiotibiais associado à crioterapia. No terceiro dia, os dois grupos foram reavaliados (AV4). 
Na semana seguinte, as avaliações foram realizadas da mesma forma que na semana anterior, invertendo apenas a intervenção nos grupos, ou seja: grupo A - alongamento estático de isquiotibiais associado à crioterapia; e grupo $\mathrm{B}$ - alongamento estático de isquiotibiais. Desta forma, visto tratar-se de um estudo cruzado, todos os voluntários receberam as duas formas de estimulação, porém em momentos distintos.

\section{Avaliação da extensibilidade muscular}

A extensibilidade dos isquiotibiais foi avaliada por meio de uma prancha desenvolvida por Brasileiro, Faria e Queiroz (19), para medição da extensibilidade de isquiotibiais. 0 indivíduo foi posicionado em decúbito dorsal, com o quadril direito sustentado a $90^{\circ}$ de flexão e o membro inferior esquerdo estendido na prancha. 0 sujeito foi fixado por faixas no nível do tórax, cintura pélvica, coxa direita e esquerda garantindo assim que as estruturas não avaliadas permanecessem estabilizadas. A altura do braço fixo de apoio para a perna direita foi adaptada de acordo com a dimensão do membro da pessoa. 0 braço móvel foi ajustado tomando-se por base o maléolo lateral da perna direita. Um goniômetro universal foi fixado ao eixo fixo e móvel da prancha para medir o ângulo extensor do joelho direito, sendo considerado $180^{\circ}$ o ângulo de extensão completa do joelho. 0 voluntário foi submetido à extensão passiva do joelho até o ponto de mínima resistência ao movimento. Para a coleta dos dados, então, foi anotado pelos pesquisadores o ângulo obtido por meio da visualização do valor apresentado no goniômetro (em graus). Após essa medida, o procedimento foi repetido no membro esquerdo.

Quanto maior a amplitude de movimento (ADM) obtida durante a extensão ativa do joelho do indivíduo, maior a extensibilidade dos músculos isquiotibiais, visto que a ADM durante essa manobra é limitada pela extensibilidade dessa musculatura, em casos que não há contratura articular ou alterações ósseas.

\section{Protocolo de aplicação de crioterapia}

Foram utilizadas bolsas de gelo contendo $1 \mathrm{~kg}$, na região posterior da coxa, durante 15 minutos.
A temperatura da bolsa foi mantida entre $0{ }^{\circ} \mathrm{C}$ e 5 ${ }^{\circ} \mathrm{C}$, avaliada por termômetro de mercúrio. Em seguida, foi realizada a aplicação do protocolo de alongamento.

\section{Protocolo de alongamento}

0 alongamento estático de isquiotibiais foi realizado em duas séries, de 30 segundos cada. 0 voluntário foi posicionado sentado e efetuou a extensão de joelhos. Em seguida, flexionou o tronco anteriormente até o ponto de desconforto. Retornou à posição inicial e após 30 segundos de repouso repetiu o alongamento.

\section{Análise estatística}

Os dados foram apresentados por estatística descritiva (média e desvio-padrão) e avaliados por estatística inferencial com uso do teste de ANOVA medidas repetidas para análise intragrupo e teste $t$ não pareado para comparar intergrupos. Na análise dentro do grupo, foi utilizado Tukey como pós-teste; em todos os casos o nível de significância aceito foi $\mathrm{p}<0,05$.

\section{Resultados}

A partir dos valores encontrados, verificou-se que os grupos foram homogêneos. A média de valores obtidos para a primeira avaliação (AV1) foi de $125,1^{\circ}( \pm 7,04)$ e na avaliação pós-alongamento (AV3) foi de $133,5^{\circ}( \pm 5,79)$. Com isso, pode-se notar que houve aumento significativo da extensibilidade dos músculos isquiotibiais, no alongamento estático (AE), quando comparada a AV1 com a AV3. Também foram observados valores de $125,1^{\circ}$ ( \pm $7,04)$ para AV1 e de $127,6^{\circ}( \pm 6,05)$ na avaliação do dia seguinte ao alongamento (AV4), verificando aumento significativo da AV1 quando comparado com a AV4. Ainda no AE, houve aumento significativo nas comparações realizadas entre a avaliação pré-alongamento (AV2), com média de $126,6^{\circ}( \pm 5,02)$, e a AV3, com média de $133,5^{\circ}( \pm 5,79)$. E entre a AV3 e AV4, houve diminuição de extensibilidade, visto que a média de valores da AV3 foi $133,5^{\circ}( \pm 5,79)$ e de AV4 foi $127,6^{\circ}( \pm 6,05)$ (Gráfico 1). 


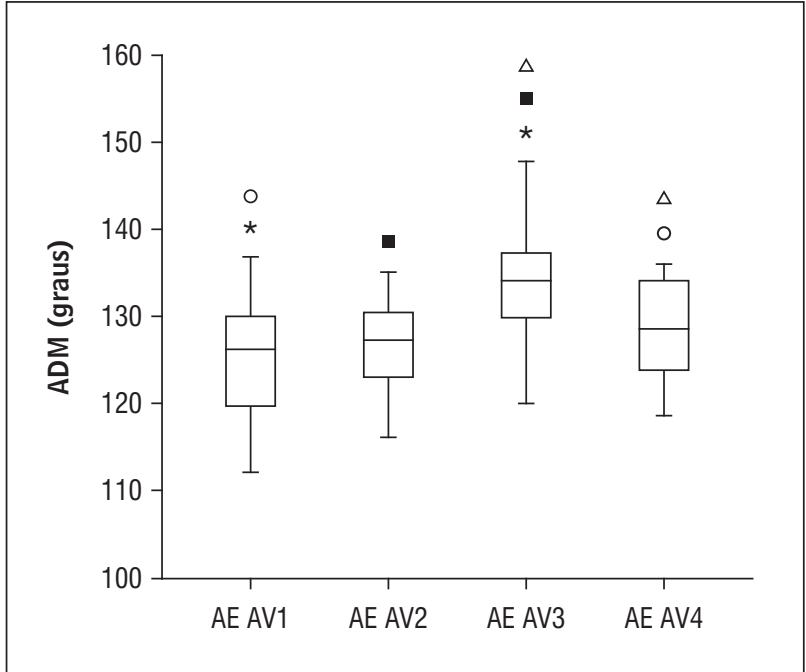

Gráfico 1 - Gráfico de representação do alongamento estático de todos os grupos.

Legenda: *Aumento significativo de extensibilidade entre a AV1 e a AV3; o Aumento significativo de extensibilidade entre a AV1 e a AV4; - Aumento significativo de extensibilidade entre a AV2 e a AV3; $\triangle$ Diminuição significativa de extensibilidade entre a AV3 e a AV4.

Fonte: Dados da Pesquisa.

Os valores encontrados no alongamento estático associado à crioterapia $(\mathrm{AE}+\mathrm{C})$ tiveram níveis de significância semelhantes nas comparações entre as mesmas avaliações do AE. A média de valores obtidos para a AV1 foi de $125,2^{\circ}( \pm 5,99)$ e na AV3 foi de $132,8^{\circ}( \pm 5,66)$. Sendo assim, pode-se notar que houve aumento significativo da extensibilidade dos músculos isquiotibiais no $\mathrm{AE}+\mathrm{C}$ quando comparada a AV1 com a AV3. Observou-se também, por meio da média de valores de $125,2^{\circ}( \pm 5,99)$ para AV1 e de $127,2^{\circ}( \pm 6,27)$ para a AV4, que houve aumento significativo da AV1 com a AV4. Ainda no AE+C, houve aumento significativo nas comparações realizadas entre a AV2, com média de $126,6^{\circ}( \pm 5,04)$, e a AV3, com média de $132,8^{\circ}( \pm 5,66)$. Já na AV3 e na AV4, houve diminuição de extensibilidade, visto que, a média de valores da AV3 foi $132,8^{\circ}( \pm 5,66)$ e de $\operatorname{AV} 4$ foi $127,2^{\circ}( \pm 6,27)$ (Gráfico 2).

Quando comparado o alongamento estático com o alongamento estático associado à crioterapia não houve diferenças significativas na extensibilidade dos músculos isquiotibiais ( $\mathrm{p}>0,05)$. Os ganhos obtidos na primeira semana foram perdidos $(\mathrm{p}<0,05)$ e, desta forma, na segunda semana os participantes continuavam como grupos homogêneos $(p>0,05)$.

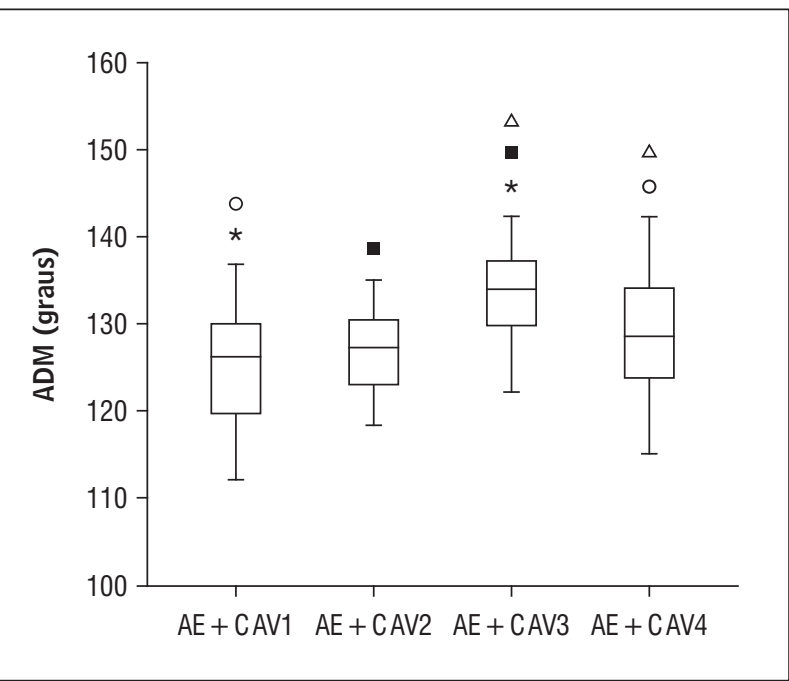

Gráfico 2 - Gráfico de representação do alongamento estático associado à crioterapia de todos os grupos.

Legenda: *Aumento significativo de extensibilidade entre a AV1 e a AV3; o Aumento significativo de extensibilidade entre a AV1 e a AV4; - Aumento significativo de extensibilidade entre a AV2 e a AV3; $\triangle$ Diminuição significativa de extensibilidade entre a AV3 e a AV4.

Fonte: Dados da Pesquisa.

\section{Discussão}

As sessões de alongamento proporcionaram ganho de extensibilidade dos músculos isquiotibiais, mas verificou-se que a aplicação de crioterapia não influenciou nesse aumento imediato da extensibilidade. $\mathrm{O}$ aumento da extensibilidade, segundo Prado et al. (34), deve-se ao remodelamento dos tecidos conjuntivo, muscular e tendíneo, os quais são acompanhados do aumento da extensibilidade, no comprimento e na resistência ao movimento passivo dinâmico.

Também foi observado que, mesmo com a perda da extensibilidade no dia seguinte ao alongamento, ainda houve ganho quando comparado com a AV1, evidenciando que o alongamento estático, independente da aplicação de agente térmicos, aumenta a extensibilidade dos músculos isquiotibiais. Estes achados confirmam alguns estudos já realizados, como o de Signori et al. (29), que verificaram o efeito de agentes térmicos sobre o aumento na extensibilidade dos isquiotibiais encurtados. Obteve-se ganho de extensibilidade, porém, os meios físicos de hipo e hipertermia não influenciaram nos resultados.

Taylor, Waring e Brashear (35) estudaram a aplicação de calor superficial ou modalidade de frio, 
seguida por alongamento estático, na eficácia do alongamento dos músculos isquiotibias. Os resultados indicaram que houve um aumento no comprimento do músculo. Entretanto, nenhuma diferença significativa foi observada entre os grupos e nem entre as modalidades térmicas.

Segundo Knight (33), com a aplicação da crioterapia, a rigidez do tecido conjuntivo aumenta e a sua extensibilidade diminui. Isso sugere que o resfriamento e o alongamento associados são prejudiciais quando se deseja aumentar a extensibilidade do tecido conjuntivo, confirmando os resultados obtidos neste estudo.

No entanto, outros estudos apresentam resultados que divergem dos valores encontrados. Brodowicz, Welsh e Wallis (31) investigaram a eficácia do crioalongamento para aumentar a extensibilidade dos músculos isquiotibiais, comparando alongamento com gelo, alongamento com calor e somente alongamento. Concluíram que a aplicação de gelo produz maior ganho na extensibilidade do que o alongamento associado ao calor ou apenas o alongamento, em curto prazo. Isso pode ser explicado baseando-se nas evidências de que a crioterapia reduz a velocidade de condução nervosa, produzindo dois efeitos importantes: diminuição da tensão muscular e da dor $(36,37)$.

Em outro estudo, realizado por Brasileiro, Faria e Queiroz (19), foi avaliada a influência do resfriamento e do aquecimento muscular local, antes das manobras de alongamento, sobre a extensibilidade dos músculos isquiotibiais. Verificou-se que os grupos tratados aumentaram a extensibilidade muscular no efeito agudo e crônico. Porém, no efeito agudo, foi observado maior ganho de extensibilidade no grupo com crioterapia.

Neste estudo, a partir das comparações realizadas, observou-se que os valores entre a AV2 e a AV4 não foram significativos. Isso pode ter ocorrido em função do estímulo de alongamento dado pelo posicionamento do indivíduo na prancha de extensibilidade durante a AV1 e a AV2.

Outros estudos podem ser desenvolvidos utilizando diferentes tempos e formas de aplicação de crioterapia. Dessa forma, será possível ampliar o conhecimento atual sobre a relação entre as modalidades térmicas e a extensibilidade dos tecidos musculares.

\section{Conclusão}

Conclui-se que houve aumento imediato da extensibilidade dos músculos isquiotibiais após a realização do alongamento estático e alongamento estático associado à crioterapia. Porém, não houve diferença significativa entre eles. Sendo assim, o uso de crioterapia não se mostrou eficaz para o ganho de extensibilidade.

\section{Referências}

1. Almeida TT, Jabur MN. Mitos e verdades sobre flexibilidade: reflexões sobre o treinamento de flexibilidade na saúde dos seres humanos. Motricidade. 2007;3(1):337-44.

2. Depino GM, Webright WG, Arnold BL. Duration of maintained hamstring flexibility after cessation of acute static stretching protocol. J Athl Train. 2000; 35(1):56-9.

3. Grego Neto A, Manffra EF. Influência do volume de alongamento estático dos músculos isquiotibiais nas variáveis isocinéticas. Rev Bras Med Esporte. 2009; 15(2):104-10.

4. Nelson RT, Bandy WD. Eccentric training and static stretching improve hamstring flexibility of high school males. J Athl Train. 2004;39(3):254-8.

5. Larsen R, Lund H, Christensen R, Roaind H, DanneskioldSamsoe B, Bliddal H. Effect of static stretching of quadríceps and hamstring muscles on knee joint position sense. Br J Sports Med. 2005;39(1):43-6.

6. Aquino CF, Gonçalves GGP, Fonseca ST, Mancini MC. Análise da relação entre flexibilidade e rigidez passiva dos isquiostibiais. Rev Bras Med Esporte. 2006; 12(4):195-200.

7. Viveiros LE, Simão R. Treinamento da flexibilidade: uma abordagem metodológica. Rev Baiana Educ Fis. 2001;2(3):20-6.

8. Almeida PHF, Barandalize D, Ribas DIR, Gallon D, Macedo ACB, Gomes ARS. Alongamento muscular: suas implicações na performance e na prevenção de lesões. Fisioter Mov. 2009;22(3):335-43.

9. Shrier I. Does stretching improve performance? A systematic and critical review of the literature. Clin J Sport Med. 2004;14(5):267-73. 
10. Gremion G. The effect of stretching on sports performance and the risk of sports injury: a review of the literature. Schweiz Z Med Traumatol. 2005; 53(1):6-10.

11. Magnusson SP, Simonsen EB, Aagaard P, DyhrePoulsen P, McHugh MP, Kjoer M. Mechanical and physical responses to stretching with and without preisometric contraction in human skeletal muscle. Arch Phys Med Rehabil. 1996;77(4):373-8.

12. Coelho LFS. O treino da flexibilidade muscular e o aumento da amplitude de movimento: uma revisão crítica da literatura. Motricidade. 2007;3(4):22-37.

13. De Deyne PG. Application of passive stretch and its implications for muscle fibers. Phys Ther. 2001; 81(2):819-27.

14. Rubini EC, Costa ALL, Gomes, PSC. The effects of stretching on strengh performance. Sports Med. 2007;37(3):213-24.

15. Young W, Behm DG. Should static stretching be used during a warmup for strength and power activities? Strength Cond J. 2002;24(6):33-7.

16. Armantrout EA, Hummel-Berry K, Underwood F, Nelson C. Physical therapist compliance with electromyography guidelines. J Neurol Phys Ther. 2008;32(4):177-85.

17. Cramer JT, Housh TJ, Weir JP, Johnson GO, Coburn JW, Beck TW. The acute effects of static stretching on peak torque, mean power output, electromyography, and mechanomyography. Eur J Appl Physiol. 2005;93(5-6):530-9.

18. Branco VR, Negrão Filho RF, Padovani CR, Azevedo CM, Alves N, Carvalho AC. Relação entre a tensão aplicada e a sensação de desconforto nos músculos isquiotibiais durante o alongamento. Rev Bras Fisioter. 2006;10(4):465-72.

19. Brasileiro JS, Faria AF, Queiroz LL. Influência do resfriamento e do aquecimento local na flexibilidade dos músculos isquiotibiais. Rev Bras Fisioter. 2007; 11(1):57-61.

20. Voigt L, Vale RGS, Abdala DW, Freitas WZ, Novaes JS, Dantas EHM. Efeitos de uma repetição de dez segundos de estímulo do método estático para o desenvolvimento da flexibilidade de homens adultos jovens. Fit Perf J. 2007;6(6):352-6.
21. Conceição MCSC, Vale RGS, Bottaro M, Dantas EHM, Novaes JS. Efeitos de quatro tempos diferentes de permanência de flexionamento estático na flexibilidade de adultos jovens. Fit Perf J. 2008;7(2): 89-92.

22. Tirloni AT, Belchior ACG, Carvalho PTC, Reis F. Efeito de diferentes tempos de alongamento na flexibilidade da musculatura posterior da coxa. Fisioter Pesq. 2008;15(1):47-52.

23. Bandy WD, Irion JM, Briggler M. The effect of time and frequency of static stretching on flexilibity of the hamstring muscles. Phys Ther. 1997;77(10):1090-6.

24. Decoster LC, Cleland J, Altieri C, Russell P. The effects of hamstring stretching on range of motion: a systematic literature review. J Orthop Sports Phys Ther. 2005;35(6):377-87.

25. Burke DG, Holt LE, Rasmussen R, MacKinnon NC, Vossen JF, Pelham TW. Effects of hot or cold water immersion and modified proprioceptiva neuromuscular facilitation flexibility exercise on hamstring length. J Athl Train. 2001;36(1):16-9.

26. Spernoga SG, Uhl TL, Arnold BL, Gansneder B. Duration of maintained hamstring flexibility after a onetime modified hold-relax stretching protocol. J Athl Train. 2001;36(1):44-8.

27. Anderson B, Burke ER. Scientific, medical and practical aspects of stretching. Clin Sports Med. 1991; 10(1):63-86.

28. Roberts JM, Wilson K. Effect of stretching duration on active and passive range of motion in the lower extremity. Br J Sports Med. 1999;33(4):259-63.

29. Signori LU, Voloski FRS, Kerkhoff AC, Brignoni L, Plentz RDM. Efeito de agentes térmicos previamente a um programa de alongamentos na flexibilidade dos músculos isquiotibiais encurtados. Rev Bras Med Esporte. 2008;14(4):328-31.

30. Enwemeka CS, Allen C, Avila P, Bina J, Konrade J, Munns S. Soft tissue thermodynamics before, during and after cold pack therapy. Med Sci Sports Exerc. 2002;34(1):45-50.

31. Brodowicz GR, Welsh R, Wallis J. Comparison of stretching with ice, stretching with heat, or stretching alone on hamstring flexibility. J Athl Train. 1996; 31(4):324-7. 
32. Shuback B, Hooper J, Salisbury L. A comparision of a self-stretch incorporating proprioceptive neuromuscular facilitation components and a therapist-applied PNF-technique on hamstring flexibility. Physiotherapy. 2004;90(3):151-7.

33. Knight KL. Crioterapia no tratamento das lesões desportivas. São Paulo: Manole; 2000.

34. Prado LG, Makarenko I, Andresen C, Kruger M, Opitz CA, Linke WA. Isoform diversity of giant proteins in relation to passive and active contractile properties of rabbit skeletal muscles. J Gen Physiol. 2005;126(5): 461-80.

35. Taylor BF, Waring CA, Brashear TA. The effects of therapeutic application of heat or cold followed by static stretch on hamstring muscle length. J Orthop Sports Phys Ther. 1995;21(5):283-6.
36. Swenson C, Sward L, Karlsson J. Cryotherapy in sports medicine. Scand J Med Sci Sports. 1996;6(4):193-200.

37. Eston R, Peres D. Effects of cold water immersion on the symptoms of exercise-induced muscle injury. J Sports Sci. 1999;(3):231-8.

Recebido: $21 / 01 / 2011$

Received: 01/21/2011

Aprovado: 07/04/2011

Approved: 04/07/2011 